\title{
A Rapid Review of the Measurement of Intrinsic Capacity in Older Adults
}

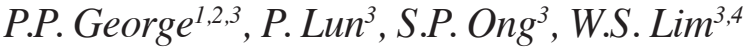

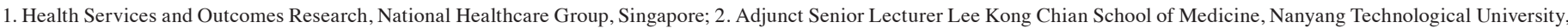

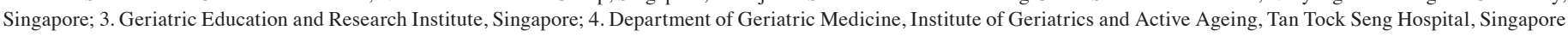 \\ Corresponding Author: Pradeep Paul George, Health Services and Outcomes Research, National Healthcare Group, Singapore,pradeep_paul_g_gunapal@nhg.com.sg
}

\begin{abstract}
OBJECTIVES: This study aims to address the knowledge gap and summarise the measurement for intrinsic capacity for the five WHO domains across different populations. It specifically aims to identify measurement tools, methods used for computation of a composite intrinsic capacity index and factors associated with intrinsic capacity among older adults.

METHODS: We performed literature review in Medline, including search terms "aged" or "elderly" and "intrinsic capacity" for articles published from 2000 - 2020 in English. Studies which assessed intrinsic capacity in the five WHO domains were included. Information pertaining to study setting, methods used for measuring the domains of intrinsic capacity, computation methods for composite intrinsic capacity index, and details on tool validation were extracted. RESULTS: Seven articles fulfilling the inclusion criteria were included in the review. Of these, the majority were conducted in community settings $(n=5)$ and were retrospective studies $(n=6)$. The most commonly used tools for assessing intrinsic capacity were gait speed test and chair stand test (locomotion); handgrip-strength and mini-nutritional assessment (vitality); Mini-Mental State Examination (cognition); Geriatric Depression Scale (GDS) and Center for Epidemiological Studies Depression Scale (CES-D) (psychological), and self-reported vision and health questionnaires (sensory). Among the tools used to operationalise the domains, we found variations and non-concordance, especially in the vitality and psychological domains, which make inter-study comparison difficult. Validated scales were less commonly used for vitality and sensory domains. Biomarkers were used for locomotion, vitality, and sensory domains. Self-reported measures were mostly used in the psychological and sensory domains. Three studies operationalised a global score for intrinsic capacity, whereby scores from the individual domains were used to create a composite intrinsic capacity index, using two approaches: a) Structural equation modelling, and b) Sub-scores for each domain which were combined either by arithmetic sum or average.

CONCLUSION: We identified considerable variations in measurement instruments and processes which are used to assess intrinsic capacity, especially among the vitality and psychological domains. A standardized intrinsic capacity composite score for clinical or community settings has not been operationalised yet. Further validation via prospective studies of the intrinsic capacity concept and computation of composite score using validated scales are needed.
\end{abstract}

Key words: Healthy aging, successful aging, elderly.

\section{Introduction}

ongruent with the global trend of population aging, the number of older people worldwide is projected to increase exponentially to over 1.5 billion in 2050 (1). It is therefore important for older adults to maintain their rights to health and enjoy good quality of life as they age. Different conceptual models have been put forward to encapsulate the notion of aging well, such as healthy ageing, active aging or successful aging (2); however, many of these models have hitherto focused on the absence of clinical diseases or the accumulation of deficits (3). Notably, there is a recent shift in focus from the perspective of mere presence or absence of disease to a function-based approach of healthy aging which is aimed at building and maintaining the functional ability of older people.

In 2015, the World Health Organization (WHO) published a world report on ageing and health and introduced the concept of intrinsic capacity (IC) to help define healthy aging (4). IC is the composite of all the physical and mental capacities of the individual and its interactions with the relevant environmental characteristics that determine the functional ability of that person, which is central for healthy ageing (5). The IC concept is aimed at measuring the capacities (as opposed to deficits) of multiple human biological systems based on body functions which are most relevant to healthy ageing $(6,7)$. Being a dynamic construct, its trajectory over time may inform clinical and public health actions, provided monitoring is contextualized at the individual or population level, respectively (8). Thus, assessment of biological age through constructs such as intrinsic capacity can enhance understanding of the functional trajectories and vulnerabilities of the individual and guide development of personalised preventive and therapeutic interventions that are tailored to the person's age, comorbidities, and preferences. Studies have also quantified the concept of IC, demonstrated it to be a powerful predictor of subsequent care dependence, and suggested a possible structure (9).

To further operationalize intrinsic capacity in a clinical context, a clinical consortium on healthy ageing was held in 2017 by the WHO to identify core components of intrinsic capacity. Five key domains were proposed: locomotion, vitality, cognitive, psychological, and sensory. Experts in the consortium proposed components and suggestions of some tools that could be used as measures for each domain (8). 
Table 1. Search strategy in Medline

\begin{tabular}{ll}
\hline Order of search & Terms \\
1 & exp Aged/ \\
2 & (elderly or senior? or «older adult?» or «older patient?» or «older people» or «older person?» or elder?).ab,ti,tw. \\
3 & 1 or 2 \\
4 & «intrinsic capacit*».ab,ti,tw. \\
5 & Healthy Aging/ or («healthy ag?ing» or «successful ag?ing»).ab,ti,tw. \\
6 & (Locomotion and Vitality and Cognition and Psychology and Sensory).ab,ti,tw. \\
7 & 5 and 6 \\
8 & 4 or 7 \\
9 & 3 and 8 \\
10 & limit 9 to (english language and yr=»2000 -Current»)
\end{tabular}

Since then, studies have shown that it is feasible to measure intrinsic capacity using commonly used measures to predict an individual's future functioning (10-17). These studies used a variety of measurements for assessing intrinsic capacity in their respective domains in different settings and populations. Although early studies support the validity of the WHO Healthy Ageing framework built around the concept of intrinsic capacity, measures of different domains are not yet standardised and vary across studies (9). There is a lack of consensus how scores from the different domains should be computed to yield a representative composite index of intrinsic capacity. Moreover, it is unclear what are the factors which are associated with low intrinsic capacity in older adults.

Therefore, this study aims to address the knowledge gap and summarise the measurement for intrinsic capacity for the five WHO domains across different populations. It specifically aims to identify measurement tools, methods used for computation of a composite intrinsic capacity index and factors associated with intrinsic capacity among older adults. A rapid review was preferred over a systematic review or a scoping review to provide timely and relevant evidence in this emerging topic that has limited literature. Our findings would identify gaps that could help inform future research agenda on the topic.

\section{Methods}

Our method is guided by the rapid review guidance document from the Agency form Healthcare Research and Quality, which draws from mainstream systematic review methodology (18). The review question was formulated and refined after discussion with stakeholders comprised of senior clinicians who are involved in the care of older adults.

\section{Search strategy}

Our search strategy was crafted with the help of a librarian using the PICOTS framework (population, interest, comparison, outcomes, timeframe, settings), where applicable. The search terms and key databases or resources were identified through consultation with geriatricians who were considered experts in the field of frailty and related areas. Table 1 presents our search strategy in Medline. The following grey literature sources were searched using simplified terms such as 'intrinsic capacity', 'healthy aging', and 'successful aging': Google Scholar, bioRxiv, medRxiv. In addition, we searched conference abstracts from 2015 to 2020 for the following "high-yield" conferences: International Conference of Frailty and Sarcopenia Research, British Geriatrics Society, American Geriatrics Society, and European Geriatrics Society. Lastly, the reference lists of retrieved key papers were also examined for articles of relevance. The above searches were performed in the month of July 2020 .

\section{Study Selection and Data Extraction}

Three reviewers (PPG, OSP, PL) screened citations using Covidence, a web-based tool. Each citation was independently screened using title and abstract by two reviewers. The relevant citations then underwent a second stage full text screening by two reviewers. A third reviewer was involved in cases of uncertainty or disagreement. Selection was based on eligibility criteria presented in table 2 and in the PRISMA flow chart, Figure 1 (19). A data extraction sheet was created to extract information on publication information, study aims, measurements performed for each domain, information on composite score and its measurement. Reviewers (PPG, OSP, PL) tested the extraction sheet using two articles to calibrate understanding of the extraction fields, prior to independent extraction of the study data. Data extraction was performed by all three reviewers and crossed checked for accuracy.

\section{Data synthesis}

Study characteristics and population demographics are presented in the form of frequencies and percentages. Information on the domain measurements and composite score were summarised and presented. Due to the significant heterogeneity in the sample populations, methods of intrinsic capacity assessment and statistical pooling of findings were not feasible. 
Table 2. Eligibility criteria

\begin{tabular}{|c|c|c|}
\hline & Inclusion criteria & Exclusion criteria \\
\hline Phenomenon of Interest & $\begin{array}{l}\text { Describes how } 5 \text { domains of intrinsic capacity } \\
\text { are measured or assessed: Locomotion, vita- } \\
\text { lity, cognition, psychology, sensory }\end{array}$ & Does not describe all 5 domains \\
\hline Setting & $\begin{array}{l}\text { Community } \\
\text { Outpatient care } \\
\text { Primary care } \\
\text { Nursing homes }\end{array}$ & Inpatient \\
\hline Study \& publication types & $\begin{array}{l}\text { Comparative study } \\
\text { Observational study } \\
\text { Clinical trial } \\
\text { Meta-analysis } \\
\text { Randomised control trial } \\
\text { Systematic review } \\
\text { Validation study } \\
\text { Evaluation study } \\
\text { Government publication } \\
\text { Technical report } \\
\text { Preprint } \\
\text { Journal article }\end{array}$ & $\begin{array}{l}\text { Editorials, } \\
\text { Opinion pieces } \\
\text { Protocols } \\
\text { All other study designs }\end{array}$ \\
\hline
\end{tabular}

\section{Results}

The literature search yielded 163 articles after removal of duplicates. Following the first round of title and abstract screening, 39 articles were eligible for the next full-text screening. Seven studies were found to fulfil the criteria for inclusion in this rapid review, Figure 1.

\section{Study characteristics}

The seven studies that fulfilled our selection criteria were published in 2019 and 2020, Table 3. Most of the studies were retrospective in nature $(n=6,86 \%)$; only one aimed to develop and validate an IC scale (14), while the rest were not designed to measure intrinsic capacity a priori. Of the seven studies, three were conducted in Europe and three in Central and South America. Six studies examined intrinsic capacity in older adults aged $\geq 60$ years. All the studies involved participants who were healthy or with co-existing medical conditions. Five studies were conducted in the community setting, whereas one study each was conducted in nursing homes and in primary care. Four studies had a sample sizes between 1000 to 10000 participants, while two studies had less than 1000 participants. Only one study had a sample size of more than 10000 participants.

\section{Measurement of intrinsic capacity by domains}

\section{Locomotion}

\section{Performance based measures}

All the seven studies employed performance-based measures for locomotion, and we have identified six measurement methods: Chair-stand, gait speed, standing balance, pick pencil, grip strength, and others, Table 4 . Most of the studies adopted at least three components as measures ( 5 out of $8,62.5 \%$ ).

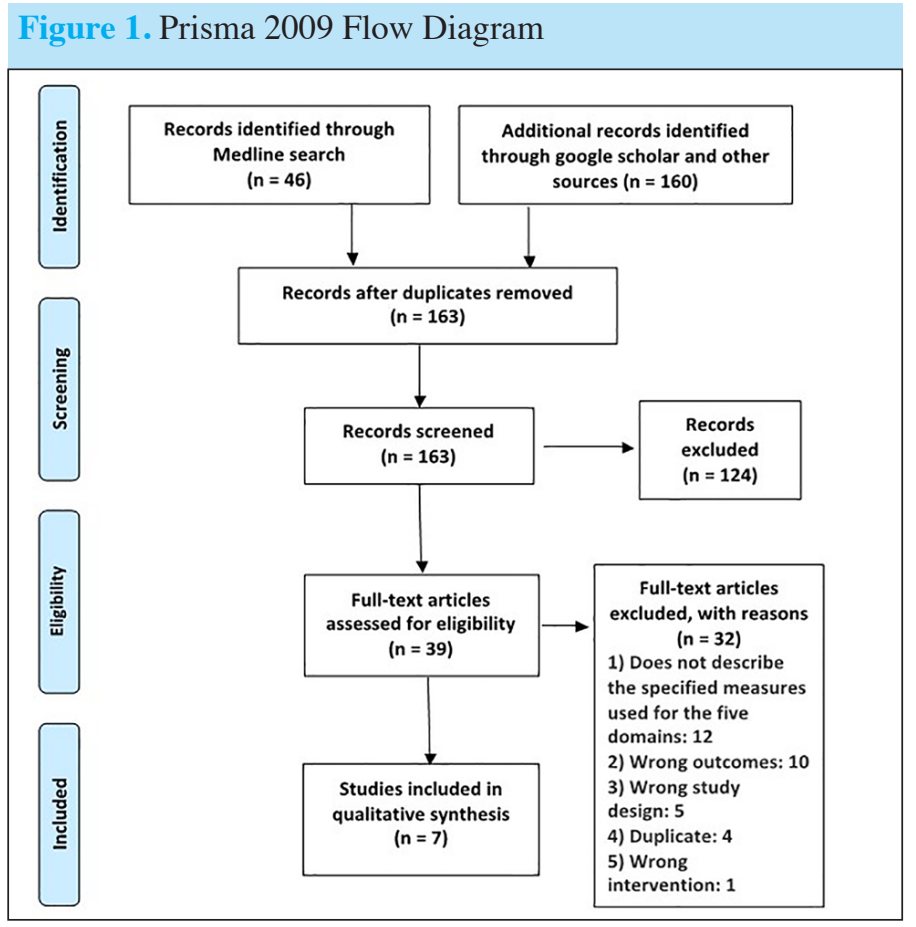

All seven studies adopted all $(10,13,16)$ or parts $(12,14$, 15) of the Short Physical Performance Battery (SPPB) (20) that consisted of chair-stand, gait speed, and standing balance. Notably, both chair-stand and gait-speed were conducted with variation across studies. For example, while time taken to sit and rise five times was commonly measured in the chair stand test (17), chair raise speed was calculated by Gutiérrez-Robledo et al (12) using the number of times an individual rose from a 
Table 3. Study characteristics and population demographics

\begin{tabular}{|l|l|l|}
\hline Publication & Categories & Percentage (n) \\
\hline Year & 2019 & $57(4)$ \\
Continent & 2020 & $43(3)$ \\
& Europe & $43(3)$ \\
\hline Study type & Central and South America & $43(3)$ \\
& Central and South America, and Asia & $14(1)$ \\
Setting & Retrospective study & $86(6)$ \\
& Prospective study & $14(1)$ \\
\hline Sample size & Primary care & $14(1)$ \\
& Community & $72(5)$ \\
\hline Age & Nursing home & $14(1)$ \\
\hline
\end{tabular}

* study included older adults

chair divided by total time taken.

For the measure of gait speed, the distance adopted among the five studies varied between 2.4 to 5 meters $(10,12,13,15$, 16) while one did not indicate the distance (12). Measurement was scored by the quicker time $(12,15)$ or mean time of two repeated tries (9) with varied definition on what was defined as having capacity (15), or graded capacity such as from worst to best $(13,16,20,21)$. For standing balance, all three studies were consistent in their methods by using hierarchical standing balance that consists of the side-by-side, semi-tandem, and fulltandem component for 10 seconds each $(10,13,16)$. Consistent with scoring protocol for SPPB, performance was rated from 0 to 4 , with 4 being the highest score.

In addition, other methods were also adopted in conjunction with SPPB or its components. Robledo et al (12) used a pickpencil test, alongside chair-rise and gait-speed, for the measure of locomotion. In another study by Gutiérrez-Robledo et al (14), handgrip strength was used together with gait speed to measure locomotion.

\section{Self-reported assessment}

While Ramírez-Vélez et al (16) adopted the three tests in SPPB, they also included other measures such as sarcopenia, prevalence of falls, impairment in activities of daily living, and mobility/disability.

\section{Vitality}

Four studies used 1 method to assess vitality and only one study used three assessment methods for this domain.

\section{Performance based measures}

Three studies assessed anthropometric measurements using body mass index $(12,13)$, abdominal circumference (13) and mid upper arm circumference (15), or body composition using phase angle derived from bioimpedance measurement (14). Two studies examined lung function using the forced expiratory volume (10) and peak flow test (12). Charles and colleagues also included the measurement of handgrip strength as an indicator of vitality (13).

\section{Self-reported assessments}

Participants were asked about any unintended weight loss and appetite in three studies $(15,16)$. Mini Nutritional Assessment $(13,15)$ as an indication of nutritional status was also employed in the assessment of vitality.

\section{Blood biomarkers}

Only one study measured biomarkers (dehydroepiandrosterone and insulin-like growth factor (10) as an assessment of vitality).

\section{Cognition}

\section{Performance based measures}

All of the studies adopted performance based measures with majority of the studies (4 of the 7; 63\%) using MMSE (12), modified MMSE $(12,16)$ and sub-components of MMSE (13) to assess cognition, Table 4. For example, Gutiérrez-Robledo 
Table 4. Measurement tools and methods used for IC domains

\begin{tabular}{|c|c|c|c|c|}
\hline Domain & Measurement tools & Description & Validation status & References \\
\hline \multirow[t]{6}{*}{ Locomotion } & Chair-rise/Chair-stand-SPPB & $\begin{array}{l}\text { Repetition of rising from chair measured in seconds (with or without using } \\
\text { arm) }\end{array}$ & Yes & $10,12,13,16,17$ \\
\hline & Gait/Walking speed-SPPB & Time taken to walk a distance $(3-10 \mathrm{~m})$ at usual pace. & Yes & $10,12-16$ \\
\hline & Standing Balance-SPPB & $\begin{array}{l}\text { Test of standing balance that progressively gets more difficult (side-by-side } \\
\text { stand, semi-tandem, full-tandem) }\end{array}$ & Yes & $10,13,16$ \\
\hline & Pick Pencil & Inverted time taken to lift a pencil from the floor & - & 12 \\
\hline & Grip Strength & Handgrip strength of the dominant hand & Yes & \\
\hline & Others & $\begin{array}{l}\text { Sarcopenia, prevalence of falls, functional impairments assessed with an } \\
\text { activities of daily living scale, mobility/disability }\end{array}$ & - & 16 \\
\hline \multirow[t]{10}{*}{ Vitality } & $\begin{array}{l}\text { Question(s) about weight loss and/ } \\
\text { or appetite }\end{array}$ & $\begin{array}{l}\text { weight loss last three month and loss of appetite; weight loss }>/=4.5 \mathrm{~kg} \text { in last } \\
\text { three months as decline in vitality }\end{array}$ & - & 16,17 \\
\hline & Peak flow test (L/min) & Not described & Yes & 12 \\
\hline & $\begin{array}{l}\text { Forced expiratory volume (FEV) } \\
\text { using spirometer }\end{array}$ & $\begin{array}{l}\text { Three readings were taken and the highest technically satisfactory measure of } \\
\text { FEV in } 1 \text { s (FEV1) was used for assessing vitality. }\end{array}$ & Yes & 10 \\
\hline & Handgrip strength (kg) & $\begin{array}{l}\text { Three measurements were taken with each hand and the maximum was } \\
\text { recorded }\end{array}$ & Yes & 13 \\
\hline & BMI & $\begin{array}{l}\text { Underweight }(<18.5) \text { or obese }(\geq 30)=0 \text {; overweight }(>25,<30)=0.5 \text { or } \\
\text { normal }(18.5-25)=1\end{array}$ & - & 12,13 \\
\hline & $\begin{array}{l}\text { Abdominal circumference (to nearest } \\
0.1 \mathrm{~cm})\end{array}$ & - & - & 13 \\
\hline & Mid-upper arm circumference & $\geq 22 \mathrm{~cm}$ & - & 15 \\
\hline & $\begin{array}{l}\text { Phase angle derived from bioimpe- } \\
\text { dance measurement }\end{array}$ & - & - & \\
\hline & Mini nutritional assessment (MNA) & Max score 30; higher score better nutrition status & Yes & 13,15 \\
\hline & Biomarkers & Dehydroepiandrosterone (DHEA) and Insulin-like growth factor (IGF-1) & - & 10 \\
\hline \multirow[t]{6}{*}{ Cognition } & MMSE & 30-point questionnaire to measure cognitive impairment & Yes (Local) & 14 \\
\hline & Modified MMSE & $\begin{array}{l}\text { Modified MMSE includes four additional questions to assess temporal and } \\
\text { spatial orientation, the ability to see relations between objects, verbal fluency, } \\
\text { and memory. }\end{array}$ & Yes & 12,16 \\
\hline & Sub-parts of MMSE & $\begin{array}{l}\text { Two parts (1) assessment of orientation ability in time and (2) memory } \\
\text { retention capacity. }\end{array}$ & Yes & 13 \\
\hline & Questions & $\begin{array}{l}\text { 1) } 3 \text { word recall 2) orientation in time and space: e.g. what is the date today? } \\
\text { where are you now? }\end{array}$ & No & 17 \\
\hline & Recall, Verbal and Letter tests & $\begin{array}{l}\text { Verbal (semantic) fluency assessed by asking participants to name as many } \\
\text { animals as they could think of in } 1 \mathrm{~min} \text {. } \\
\text { Delayed verbal memory assessed using lists of nouns presented aurally. } \\
\text { Attention assessed using a letter cancellation task. }\end{array}$ & $\begin{array}{l}\text { Yes (Verbal fluency, } \\
\text { face validity) }\end{array}$ & 10 \\
\hline & $\begin{array}{l}\text { Community Screening Instrument for } \\
\text { Dementia (CSI-D) }\end{array}$ & $\begin{array}{l}\text { CSI-D is a screening instrument for dementia has two components, a cognitive } \\
\text { test for non-literate/literate populations and an informant interview regarding } \\
\text { performance in everyday living. }\end{array}$ & Yes & 15 \\
\hline \multirow[t]{11}{*}{ Psychological } & Self-reported depressive symptoms & $\begin{array}{l}\text { Uses } 2 \text { questions: } 1 \text {. Feeling down, depressed, or hopeless? 2. Little interest or } \\
\text { pleasure in doing things? }\end{array}$ & Not mentioned & 17 \\
\hline & Geriatric Depression Scale (GDS-15) & 15-item of self-report measure of depression in older adults & Yes & 12 \\
\hline & Geriatric Depression Scale & Not described & Yes & 16 \\
\hline & $\begin{array}{l}\text { EuroQol-5D-use of } \\
\text { «anxiety/depression" item }\end{array}$ & $\begin{array}{l}\text { 3-point Likert scale: ( } 1=\text { "I am not anxious or depressed", } 2 \text { = "I am modera- } \\
\text { tely anxious or depressed", } 3 \text { = "I am extremely anxious or depressed"). }\end{array}$ & Yes & 13 \\
\hline & EURO-D depression scale & 12-item depressive symptoms scale for older adults & Yes & 15 \\
\hline & $\begin{array}{l}\text { Center for Epidemiological Studies } \\
\text { Depression scale-CES-D }\end{array}$ & Self-report depression scale (use of 7 or 8 -item) & Yes & 10,14 \\
\hline & $\begin{array}{l}\text { Center for Epidemiological Studies } \\
\text { Depression scale-CES-D (fatigue) }\end{array}$ & $\begin{array}{l}\text { Self-report on fatigue («I felt that everything I did was an effort» and «I could } \\
\text { not get going during the past week») }\end{array}$ & Yes & 13 \\
\hline & Self-report sleep disturbance & $\begin{array}{l}\text { The frequency of delay in falling asleep, inability to stay asleep, waking up } \\
\text { tired and disturbed sleep in the previous month }\end{array}$ & Yes & 10 \\
\hline & Self-report life satisfaction & Question asked: «In general, how do you feel about your life?» & Yes* & 12 \\
\hline & Self-report locus of control & measured with eight items, that were summed & Yes* & 12 \\
\hline & Self-report Social participation & $\begin{array}{l}\text { Average number of hours dedicated in the last } 12 \text { months to the following } \\
\text { activities: providing help to other adults, church, childcare, civic activities, } \\
\text { watching TV, sports, daily tasks, recreational activities }\end{array}$ & Not mentioned & 12 \\
\hline
\end{tabular}


Table 4. Measurement tools and methods used for IC domains (continued)

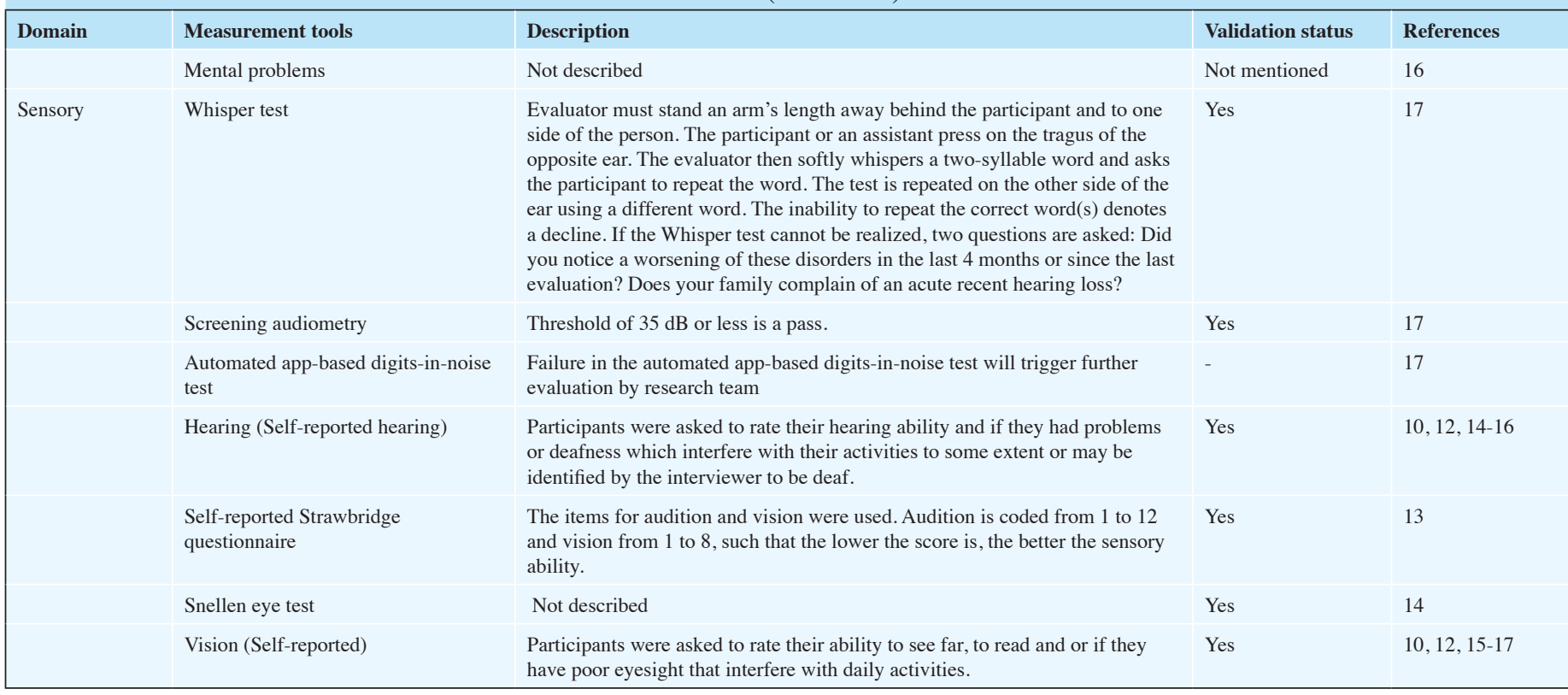

Not described; * Validated locally

et al (12) used a modified MMSE to assess cognition. Scores were standardized and stratified by education to define 3 cut-off points (severe deterioration $[>2.5 \mathrm{SD}]$, slight deterioration $[\geq 1.5$ to $\leq 2.5]$, optimal $[<1.5 \mathrm{SD}])$.

Three studies used other methods to assess cognition. One study used questions on orientation in time, space, and recall (17), one used using neuropsychological tests such as delayed recall, animal category fluency and letter cancellation (10), and Community Screening Instrument for Dementia (CSI-D) (14). In the latter study, scores in the cognitive tests were used as measures of the respective cognitive function, namely memory, executive function, and processing speed, respectively. Lastly, one study used a cut-off of $\geq 29.5$ on the CSI-D to assess cognitive capacity, with scores below that threshold indicating 'probable dementia' (15).

\section{Psychological}

\section{Self-reported assessment}

All the studies used self-reported assessment scales or questions to assess depressive symptoms. These studies used a variety of tools to measure depression, with some using tools tailored specifically for older adults. The most commonly used scales were the Geriatric Depression Scale (GDS) (12) and the Center for Epidemiological Studies Depression scale (CESD) $(10,14)$. Other studies utilized the EURO-D depression scale (15), a single item from EuroQOL-5D on «anxiety or depression» to identify presence and severity of anxiety or depression (13), and two questions to assess presence of depressed or hopeless feelings, and the experience of no interest or pleasure (17).

In addition to depressive symptoms, other symptoms in the psychological domain that were assessed include fatigue by using two questions from CES-D (13), sleep disturbance (10), and mental health problems (16). Gutiérrez-Robledo et al (12) included the most components for the psychological domain, measuring life satisfaction, locus of control, and social participation, as well as symptoms of depression.

\section{Sensory}

\section{Performance based measures}

Performance tests to assess hearing included the whisper test where the researcher whispers a two-syllabus word into the participants' ears (16); audiometry test (16); or automated app-based digits-in-noise test (17). With regards to performance tests to assess vision, the Snellen eye test was adopted in only one study (14).

\section{Self-reported assessments}

All studies assessed vision and hearing for the sensory domain using self-reported questionnaires to assess either vision and/or hearing, Table 4 . Only two studies $(10,13)$ used self-reported questions (such as the Strawbridge questionnaire) to assess deficits in hearing or vision. Some of the questions related to vision included the ability to see far, to read (10, $12,17)$ and poor eyesight that interfered with daily activities (15). Similarly, participants were asked about their hearing in general (12) and if they have hearing problems or deafness that interfered with their daily activities (15). 


\section{Methods used for estimating composite intrinsic capacity score}

Three studies derived a composite intrinsic capacity score $(10,12,14)$. In two of these studies $(12,14)$, each domain of the 5 components of IC was further divided into three categories, assigning a numerical score as follows: optimal $=0$, mild impairment $=1$ and severe impairment $=2$. Scores of each domain was then summed to yield a complete intrinsic capacity index, ranging from zero (worst IC possible) to ten (optimal intrinsic capacity possible). The intrinsic capacity composite score for one study showed low internal consistency (Cronbach alpha $=0.49$ ), whereas factor analysis demonstrated substantial clustering of each domain to a single factor (14).

Beard et al (10) used the following tests to measure intrinsic capacity for the five domains: gait speed, chairstand and standing balance tests of the SPPB (Locomotion); dehydroepiandrosterone (DHEA) and insulin-like growth factor (IGF-1), and forced expiratory volume (FEV) (Vitality); verbal fluency, delayed verbal memory, and letter cancellation tasks (Cognition); depression using the CES-D scale and sleep disturbances (Psychological); and self-reported hearing and visual impairment (Sensory). A bi-factor model that represented a pure measure of the underlying latent trait of interest, after controlling for all five specific subfactors, was used to estimate the individual factor score. The summary score of intrinsic capacity and specific subfactors showed good construct validity and displayed significant differences between sex, age, disease, and functional status.

\section{Factors and outcomes associated with intrinsic capacity}

Studies reported a decline in intrinsic capacity with increasing age $(12,14,15)$. In women, the odds of having a lower intrinsic capacity score are $72 \%$ higher than among men (12). Socioeconomic condition (SEC) level and intrinsic capacity are positively associated, such that the higher the SEC level, the higher the intrinsic capacity score (12). In the domains of continence, hearing, and vision, declines in capacity were more pronounced after the age of 80 (15).

A unit increase in balance performance and in nutrition score decreased the probability of death by $12 \%$ and $4 \%$ respectively (13). The risk of falling decreases, when there is a one-unit increase in balance performance and in nutrition score. Low scores in nutrition are associated with a higher probability of autonomy decline (13). Declines in cognition and continence are strongly associated with incident dependence, and declines in psychological capacity, locomotion, and nutrition are moderately associated, while sensory capacities (vision and hearing) are not associated with incident dependence (15). Participants with optimal handgrip strength had better intrinsic capacity, while gender-stratified analyses revealed that men with optimal handgrip strength had a lower risk of hospitalization (16).

Multimorbidity has an independent direct relationship with incident loss of basic activities of daily living (ADLs), but not instrumental activities of daily living (IADLs). However, a greater proportion of the indirect effect on incident loss of ADLs and IADLs was mediated by intrinsic capacity than by multimorbidity (10). When compared with those without co-morbidity, the odds of having a significant decrease in intrinsic capacity score rose significantly (51\%) among those with three or more conditions (12).

\section{Discussion}

Consistent with the ongoing shift in conceptual focus of healthy aging from a disease or deficit-centric to a functioncentric model, the concept of intrinsic capacity was conceived to define a composite of all physical and mental capacities of an individual, and which interacts with the environment to determine functional ability (4). Our rapid review aimed at providing timely understanding of how measurements of the domains were operationalized to date, and the factors and outcomes associated with intrinsic capacity. Timely review of this information would facilitate ongoing pilot implementation of ICOPE guidelines worldwide, and facilitate better understanding of individual functional trajectories and vulnerabilities during a catastrophic event such as the current Covid-19 pandemic (22). Although a few studies have documented the measurement of intrinsic capacity and its association with health outcomes, not much is known about the collective body of evidence. We focused on studies that assessed all five intrinsic capacity domains, unlike a recent critical literature review by Gonzales and colleagues (9) which included studies with any of the five domains. Both reviews examined the assessment of intrinsic capacity, and the tools used to measure the domains. However, our review goes beyond Gonzalez et al (9) to document the association between intrinsic capacity with age, gender, socio-economic status, nutrition, mortality, falls risk, hospitalisation, ADLs, IADLs and multimorbidity.

Among the included studies, the majority were retrospective studies using data collected in the community (fig 3 ) and hence, were not primarily aimed to assess intrinsic capacity. Most commonly used tools for assessing intrinsic capacity were gait speed test and chair stand test (locomotion), grip-strength and mini-nutritional assessment (Vitality), mini-mental state examination (Cognition), Geriatric Depression Scale (GDS) or Centre for Epidemiological Studies Depression scale (CES-D), and self-reported vision and health questionnaires (Sensory). However, we found heterogeneity and low concordance in operationalization of some of the domain measurements, particularly the vitality and psychological domains, which would make cross-study comparisons difficult. In addition, even within the same tool, there were variations among the studies. For example, in the measure of gait speed in the Short Physical Performance Battery test, the distance executed in the studies and calculation of the speed were varied $(10,12,13,15,16)$. Reasons for the heterogeneity and low concordance could be in part due to the fact that most studies utilized retrospective data that were designed to measure other aims, Table 3 . Hence, they were not primarily aimed to assess intrinsic capacity per se. 
Only one on-going study, the INSPIRE-ICOPE-CARE (16), set out to measure the five domains of intrinsic capacity a priori.

Of all the domains, vitality is the most heterogeneous in its operationalization. It was assessed using different measures such as body composition, lung function, nutritional status, physical strength, specific molecular biomarkers and use of health questions related to weight loss and appetite. A similar observation in the vitality domain was also reported in a systematic review (9). Vitality has been proposed as the key domain in the construct of intrinsic capacity that drives the other domains (23). In this iteration, cellular level characteristics as well as the contribution of higher physiologic systems are included within the vitality construct; when the accumulation of accumulated deficits in these systems reach a certain point, they become manifest in the overt losses of capacity that are commonly associated with ageing (24). Using this conceptual framework, a total measure of vitality may thus capture an individual's 'biological age' (10). Therefore, it is important to have a clearer illumination of the concepts pertaining to vitality and to achieve consensus on its appropriate measurement and weightage.

Validity and reliability are two key attributes to consider when selecting instruments for measuring any health outcome (25). In our review, we found that most of the instruments were validated. Performance-based tests and blood molecular biomarkers were used for locomotion and vitality domain, respectively. Self-reported measures were mostly used for psychological and sensory domains, which may increase social desirability bias and recall bias $(26,27)$ as compared to the other domains. On the other hand, none of the studies employed self-reported measures in the cognitive domain; the complementary use of validated scales or questions that evaluate subjective memory complaints such as the AD8 may be less susceptible to ceiling effects or educational bias in bedside mental status tests such as the MMSE $(28,29)$. Validity and reliability of intrinsic capacity composite score can also be further improved by using measurement tools less prone to bias (30) and using appropriate weightage for the different intrinsic capacity domains.

Of the seven studies identified in our review, only three operationalised a global intrinsic capacity score. Scores from the different domains were combined to create a global intrinsic capacity index using two approaches. Beard and colleagues (10) used an integrated approach to estimate factor score for each study participant, while the others $(12,14)$ used a summation of individual domain score to compute the intrinsic capacity index. Though the three studies provided information on the measurement of intrinsic capacity for the five WHO domains, information on summation methods and weightage were not adequately presented in the studies. A composite intrinsic capacity which is a summative measure of individual domain scores for the five WHO domains can be used, akin to the growth charts used to chart development in children, so that if population gender-specific norms are established, any significant deviation could be detected early with the early institution of treatment (31). The individual domain scores would provide information on one's affected intrinsic capacity domains, which could guide interventions.
One the other hand, the remaining studies utilized information from each individual domain for feedback (13, 15-17). Among this group of studies, variations on the measure of decline were observed. Three studies measured the domains by either using one or at least two measures, of which the average results in the measures were used to determine capacity or decline in that domain (15-17). The fourth study in a nursing home population used between two to four measures for each domain and identified balance performance and nutrition (as measured by the MNA) to predict adverse health outcomes (13). Three of these studies were retrospective in nature, with goals to identify associations of the domains with adverse outcomes and dependence $(13,15,16)$. The only prospective study was aimed at flagging decline, which would alert the need for further assessments and interventions (17). The overall goals in the studies were to measure and identify ways to use the five domains to detect one's intrinsic capacity, which might help inform actions and interventions in clinical practice and healthcare systems. However, our current review highlighted that the current adopted methods to measure the domains were heterogenous and varied, which would present challenges in validating the use of IC across different populations.

\section{Limitations}

The study had few inherent limitations of a rapid review. It was conducted to provide evidence to stakeholders within a short time frame. In view of the time constraint, trade-offs in methodological choices were made, such as restricting the research to Medline and English publications. This may have resulted in omission of relevant data and left the review susceptible to selection and language bias. Despite these limitations, the review provided a timely synthesis of the evidence on the measurement for intrinsic capacity for the five WHO domains across different populations.

\section{Conclusion}

Intrinsic capacity in the context of healthy ageing has been measured using self-reported questionnaire, performancebased tests, and laboratory investigations. There is variation in measurement process or tools used for assessing intrinsic capacity domains, particularly in the vitality and psychological domains. A standard intrinsic capacity score for clinical or community-based has not been operationalised yet. Further validation of the intrinsic capacity concept and computation of composite intrinsic capacity score using validated scales is needed.

Acknowledgement: We would like to express our sincere appreciation to Ms Yasmin Lynda Munro at the Nanyang Technological University, Lee Kong Chian School of Medicine Library, for helping us with the Medline database search. In addition, we would like to thank the valuable feedback from the senior management at the Geriatric Education and Research Institute: A/P Dr Ding Yew Yoong, Dr Wong Chek Hooi, Dr Chong Mei Sian, A/Prof Wee Shiou Liang, A/Prof James Low. .

Conflicts of Interest: None declared for all authors. 


\section{References}

1. Nations U. World population ageing 2019: Highlights. World population ageing. 2019;

2. Peterson JR, Baumgartner DA, Austin SL. Healthy ageing in the far North: perspectives and prescriptions. International Journal of Circumpolar Health. 2020/01/01 2020;79(1):1735036. doi:10.1080/22423982.2020.1735036

3. Belloni G, Cesari M. Frailty and intrinsic capacity: two distinct but related constructs. Frontiers in Medicine. 2019;6:133.

4. Organization WH. World report on ageing and health. World Health Organization; 2015.

5. Beard J. O cer AMPH, de Carvalho IAMD, Sadana RS, Pot AMP, Michel J-PMD, Lloyd-Sherlock PP, Epping-Jordan JEP, Peeters GMEEP, Mahanani WRM et al: The World report on ageing and health: a policy framework for healthy ageing. Lancet, The. 2016;387(10033):2145-2154.

6. Cesari M, Araujo de Carvalho I, Amuthavalli Thiyagarajan J, et al. Evidence for the domains supporting the construct of intrinsic capacity. The Journals of Gerontology: Series A. 2018;73(12):1653-1660.

7. Chatterji S, Ustün BL, Sadana R, Salomon JA, Mathers CD, Murray CJ. The conceptual basis for measuring and reporting on health. Global Programme on Evidence for Health Policy Discussion Paper. 2002;45

8. Organization WH. WHO clinical consortium on healthy ageing: topic focus: frailty and intrinsic capacity: report of consortium meeting, 1-2 December 2016 in Geneva, Switzerland. 2017.

9. Gonzalez-Bautista E, Andrieu S, Gutiérrez-Robledo LM, García-Chanes RE, De Souto Barreto P. In the Quest of a Standard Index of Intrinsic Capacity. A Critical Literature Review. The journal of nutrition, health \& aging. 2020/05/21 2020; doi:10.1007/ s12603-020-1394-4

10. Beard JR, Jotheeswaran A, Cesari M, de Carvalho IA. The structure and predictive value of intrinsic capacity in a longitudinal study of ageing. BMJ open. 2019;9(11)

11. Guyonnet S, Rolland Y, Takeda C, et al. The INSPIRE Bio-resource Research Platform for Healthy Aging and Geroscience: Focus on the Human Translational Research Cohort (The INSPIRE-T Cohort). The Journal of Frailty \& Aging. 2020:1-11.

12. Gutiérrez-Robledo LM, García-Chanes R, Pérez-Zepeda M. Allostatic Load as a Biological Substrate to Intrinsic Capacity: A Secondary Analysis of CRELES. The journal of nutrition, health \& aging. 2019;23(9):788-795.

13. Charles A, Buckinx F, Locquet M, et al. Prediction of adverse outcomes in nursing home residents according to intrinsic capacity proposed by the World Health Organization. The Journals of Gerontology: Series A. 2020;75(8):1594-1599.

14. Luis Miguel Gutiérrez-Robledo REG-C, Oscar Rosas-Carrasco. Adaptation and Validation Of A Scale To Assess Intrinsic Capacity In Mexican Community-Dwelling Elderly Adults - Abstract: Symposia, Conferences, Oral communications: 10th International Conference on Frailty, Sarcopenia Research and Geroscience (ICFSR), March 11-13, 2020, Toulouse - France. Conference Abstract. The Journal of Frailty \& Aging. 2020 2020;9(S1):S1-S45. doi:10.14283/jfa.2020.8

15. Prince $\mathrm{MJ}, \mathrm{Acosta} \mathrm{D}$, Guerra $\mathrm{M}$, et al. Intrinsic capacity as a framework for Integrated Care for Older People (ICOPE); insights from the 10/66 Dementia Research Group cohort studies in Latin America, India and China. medRxiv. 2019:19006403.
16. Ramírez-Vélez R, Correa-Bautista JE, García-Hermoso A, Cano CA, Izquierdo M. Reference values for handgrip strength and their association with intrinsic capacity domains among older adults. Journal of cachexia, sarcopenia and muscle. 2019;10(2):278-286

17. Tavassoli N, Piau A, Berbon C, et al. Framework Implementation of the Inspite ICOPE-Care program in collaboration with the World Health Organisation (WHO) in the Occitania region.

18. Hartling L, Guise J-M, Hempel S, et al. Fit for purpose: perspectives on rapid reviews from end-user interviews. Syst Rev. 2017;6(1):32-32. doi:10.1186/s13643-017-0425-7

19. Moher D, Shamseer L, Clarke M, et al. Preferred reporting items for systematic review and meta-analysis protocols (PRISMA-P) 2015 statement. Syst Rev. 2015;4(1):1.

20. Guralnik JM, Simonsick EM, Ferrucci L, et al. A short physical performance battery assessing lower extremity function: association with self-reported disability and prediction of mortality and nursing home admission. Journal of gerontology. 1994;49(2):M85-M94.

21. Gómez JF, Curcio C-L, Alvarado B, Zunzunegui MV, Guralnik J. Validity and reliability of the Short Physical Performance Battery (SPPB): a pilot study on mobility in the Colombian Andes. Colombia medica. 2013;44(3):165-171.

22. Lim WS, Liang CK, Assantachai P, et al. COVID-19 and Older People in Asia: AWGS Calls to Actions. Geriatrics \& Gerontology International. 2020;

23. Stuck AE, Walthert JM, Nikolaus T, Büla CJ, Hohmann C, Beck JC. Risk factors for functional status decline in community-living elderly people: a systematic literature review. Social science \& medicine. 1999;48(4):445-469.

24. World Health O. WHO clinical consortium on healthy ageing 2017: focus: development of comprehensive assessments and care plans: report of consortium meeting, 21-22 November 2017 in Geneva Switzerland. 2018. 2018. https://apps.who. int/iris/handle/10665/272375

25. Souza ACd, Alexandre NMC, Guirardello EdB. Propriedades psicométricas na avaliação de instrumentos: avaliação da confiabilidade e da validade. Epidemiologia e Serviços de Saúde. 2017;26:649-659.

26. Althubaiti A. Information bias in health research: definition, pitfalls, and adjustment methods. J Multidiscip Healthc. 2016;9:211-217. doi:10.2147/JMDH.S104807

27. Biemer PP, Groves RM, Lyberg LE, Mathiowetz NA, Sudman S. Measurement errors in surveys. vol 173. John Wiley \& Sons; 2011

28. Galvin JE, Roe CM, Xiong C, Morris JC. Validity and reliability of the AD8 informant interview in dementia. Neurology. 2006;67(11):1942-1948.

29. Lim WS, Chong MS, Sahadevan S. Utility of the clinical dementia rating in Asian populations. Clinical medicine \& research. 2007;5(1):61-70.

30. Sullivan GM. A primer on the validity of assessment instruments. J Grad Med Educ. 2011;3(2):119-120. doi:10.4300/JGME-D-11-00075.1

31. Sanchez-Rodriguez D, Annweiler C, Gillain S, Vellas B. Implementation of the Integrated Care of Older People (ICOPE) App in Primary Care: New Technologies in Geriatric Care During Quarantine of COVID-19 and Beyond. The Journal of Frailty \& Aging. 2020:1-2. doi:10.14283/jfa.2020.24 